This is the peer reviewed version of the following article: Dr. Shaohua Liu, Dr. Jian Zhang, Dr. Renhao Dong, Dr. Pavlo Gordiichuk, Dr. Tao Zhang, Dr. Xiaodong Zhuang, Prof. Yiyong Mai, Prof. Feng Liu, Prof. Andreas Herrmann, Prof. Xinliang Feng. Two-Dimensional Mesoscale-Ordered Conducting Polymers. Angew.Chem.Int. Ed. 2016, 55, 12516 -12521, which has been published in final form at https://doi.org/10.1002/anie.201606988. This article may be used for non-commercial purposes in accordance with Wiley Terms and Conditions for Self-Archiving. 


\title{
Two-Dimensional Mesoscale-Ordered Conducting Polymers
}

\author{
Shaohua Liu, Jian Zhang, Renhao Dong, Pavlo Gordiichuk, Tao Zhang, Xiaodong Zhuang, Yiyong Mai, \\ Feng Liu, Andreas Herrmann, and Xinliang Feng*
}

\begin{abstract}
Despite the availability of numerous two-dimensional (2D) materials with structural ordering at the atomic or molecular level, direct construction of mesoscale-ordered superstructures within a 2D monolayer remains an enormous challenge. Here, we report the synergic manipulation of two types of assemblies in different dimensions to achieve 2D conducting polymer nanosheets with structural ordering at the mesoscale. The supramolecular assemblies of amphipathic perfluorinated carboxylic acids and block co-polymers serve as 2D interfaces and meso-inducing moieties, respectively, which guide the polymerization of aniline into $2 \mathrm{D}$, freestanding mesoporous conducting polymer nanosheets. Grazingincidence small-angle $\mathrm{X}$-ray scattering combined with various microscopy demonstrates that the resulting mesoscale-ordered nanosheets have hexagonal lattice with $d$-spacing of about $30 \mathrm{~nm}$, customizable pore sizes of 7-18 nm and thicknesses of $13-45 \mathrm{~nm}$, and high surface area. Such template-directed assembly produces polyaniline nanosheets with enhanced $\pi-\pi$ stacking interactions, thereby resulting in anisotropic and record-high electrical conductivity of approximately $41 \mathrm{~S} \mathrm{~cm}^{-1}$ for the pristine polyaniline nanosheet based film and approximately $188 \mathrm{~S} \mathrm{~cm}^{-1}$ for the hydrochloric acid-doped counterpart. Our moldable approach creates a new family of mesoscale-ordered structures as well as opens avenues to the programmed assembly of multifunctional materials.
\end{abstract}

Two-dimensional (2D) ultrathin materials like graphene have attracted increasing attention in chemistry, materials science, nanotechnology and biology due to their fascinating optical, electrical and magnetic properties resulting from the reduction of dimensions. ${ }^{[1]}$ To pave the way for the rapid development of $2 \mathrm{D}$ materials, it is essential to overcome limitations with respect to

[*] Dr. S. Liu, Dr. J. Zhang, Dr. R. Dong, Dr. T. Zhang, Dr. X. Zhuang, Prof. X. Feng

Center for Advancing Electronics Dresden (cfaed) \&

Department of Chemistry and Food Chemistry

Technische Universität Dresden, 01062 Dresden, Germany

E-mail: xinliang.feng@tu-dresden.de

Dr. P. Gordiichuk and Prof. A. Herrmann

Polymer Chemistry and Bioengineering Group, Zernike Institute for

Advanced Materials, University of Groningen

Nijenborgh 4, Groningen 9747 AG, The Netherlands

Dr. X. Zhuang and Prof. Y. Mai

School of Chemistry and Chemical Engineering

Shanghai Jiao Tong University

800 Dongchuan Road, 200240, Shanghai, P. R. China

Prof. F. Liu

State Key Laboratory for Mechanical Behavior of Materials

Xi'an Jiaotong University, 710049, Xi'an, P. R. China

Supporting information for this article is given via a link at the end of the document. synthetic accessibility and structural control. As part of the ongoing efforts to develop new techniques, such as mechanical cleavage, fluid exfoliation and chemical vapor deposition, a series of inorganic atomic-layered materials, including transition metal sulfides, nitrides and oxides, have been successfully fabricated. $\left.{ }^{[1 a}, 1 d, 2\right]$ The explorations of surface-mediated synthesis on inert crystalline surfaces, liquid-air and liquid-liquid interfaces have led to new 2D soft organic and polymeric networks with periodic structures at the molecular level. ${ }^{[3]}$

The creation of mesoscale-ordered superstructures is an alluring goal and represents a key step toward many potential applications. ${ }^{[4]}$ 2D mesoscale-ordered nanosheets with topologically in-plane repeat units, especially composed of welldefined, periodic, 2-50 nm mesopores/mesochannels, would provide materials with outstanding and exotic functions. ${ }^{[4 d, 5]}$ Although the recently well-established self-assembly techniques provide possibilities for producing diverse architectures, bottomup construction of 2D mesoscale-ordered superstructures remains undeveloped due to the lack of control over the sophisticated molecular building blocks. ${ }^{[3 a-c, 6]}$

In this study, we report the construction of 2D, free-standing conducting polymer nanosheets with mesoscale ordering by synergic manipulation of two types of assemblies in different dimensions. First, amphiphilic molecules of perfluorocarboxylic acids (PFCA) and polystyrene- $b$-poly(ethylene oxide) (PS- $b$ PEO) diblock copolymers self-assemble into 2D free-standing lipid bilayers and spherical micelles in solution, respectively. Upon direct mixing of the above preformed self-organized assemblies, they co-assemble into refined hierarchical superstructures by monolayer close-packing of the spherical micelles on both interfaces of the free-standing lipid bilayers, which further act as co-templates to confine the growth of polyaniline (PANI) into ultrathin mesoscale-ordered porous nanosheets. Grazing-incidence small-angle X-ray scattering (GISAXS) combined with microscopy techniques reveals that the resulting mesoporous polyaniline nanosheets feature an ordered hexagonal structures with $d$-spacing of about $30 \mathrm{~nm}$, controlled pore sizes $(7-18 \mathrm{~nm})$ and thicknesses (13-45 nm), as well as high surface area $\left(85 \mathrm{~m}^{2} \mathrm{~g}^{-1}\right)$. Such template-directed assembly results in a compact arrangement of PANI polymer backbones with enhanced $\pi-\pi$ stacking along the in-plane directions, thereby providing the resulting mesoporous PANI (mPANI) nanosheets with anisotropic electrical conductivity. Meanwhile, record-high electrical conductivity of $41 \mathrm{~S} \mathrm{~cm}^{-1}$ is also achieved for the pristine mPANI nanosheet based films (as-made samples without any further treatment), which can be further increased to $188 \mathrm{~S} \mathrm{~cm}^{-1}$ upon hydrochloric acid doping.

Conducting polymers (such as polyaniline and polypyrrole) containing a conjugated $\pi$-electron backbone exhibit interesting optical and electrical properties and have been extensively utilized in energy storage systems, sensors and electronic 
devices. ${ }^{[7]}$ To achieve the bottom-up fabrication of $2 \mathrm{D}$ conducting polymer nanostructures with controlled ordering at the mesoscale, we first conceived a synergistic self-assembly route through a monolayer, close-packing arrangement of BCP spherical micelles on both interfaces of in-situ generated lipid lamellas. After systematic investigation of various self-assembly systems based on low-molecular-weight lipids in solution, we found that amphiphilic PFCA molecules with rigid hydrophobic fluoroalkyl chains can self-assemble to yield stable ultrathin bilayer structures, as schematically illustrated in Figure 1a-1. Cryogenic transmission electron microscopy (cryo-TEM) and solution-based fluid atomic force microscopy (AFM) measurements unambiguously confirmed their self-assembly in ethanol $/ \mathrm{H}_{2} \mathrm{O}$ solution. A typical cryo-TEM image in Figure 1a-2 shows that the assembly of 18-carbon PFCA molecules (denoted as $\mathrm{C}_{18}-\mathrm{PFCA}$, similar for $\mathrm{C}_{12}$-PFCA and $\mathrm{C}_{14}-\mathrm{PFCA}$ ) form transparent and thin nanosheets. A fluid AFM observation in tapping mode (Fig. 1a-3) revealed that the resulting nanosheets have smooth surfaces with a uniform thickness of $3.8 \pm 0.12 \mathrm{~nm}$, suggesting a typical bilayer structure derived from densely stacked lipid chains of PFCA. The interior of the sandwiched structure is composed of hydrophobic lipid chains, whereas the polar heads consisting of the carboxylic acid groups are located at the exterior surfaces and are exposed to the surrounding solution. ${ }^{[8]}$ Therefore, the ultrathin $\mathrm{C}_{18}$-PFCA nanosheets can serve as free-standing $2 \mathrm{D}$ interfaces, in which the abundant carboxylic acid groups guide the 2D self-assembly of other functional molecules or nanoparticles via hydrogen bonding.
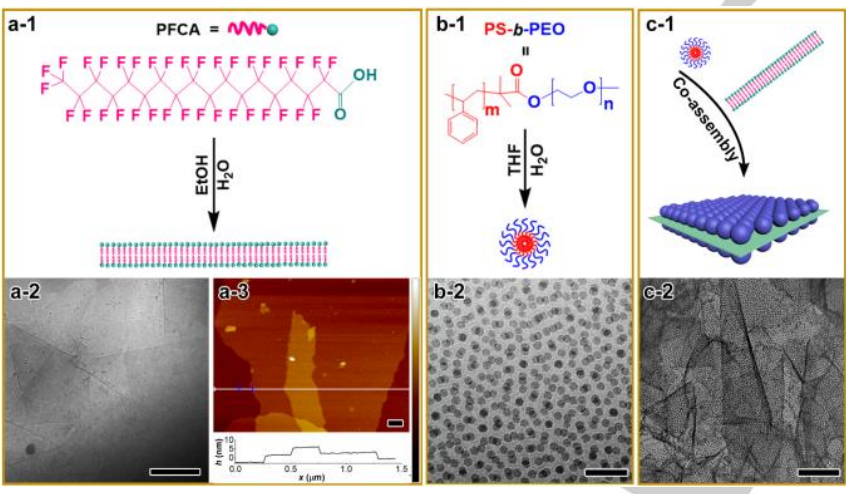

Figure 1. Manipulating molecular building blocks for cooperative selfassembly. (a-1) Schematic formation of 2D PFCA nanosheets with bilayer structure derived from the densely stacked lipid chains of PFCA in an $\mathrm{EtOH}$ and $\mathrm{H}_{2} \mathrm{O}$ solvent mixture at room temperature. (a-2) The corresponding cryoTEM images presented the apparent nanosheet morphology of self-assembled PFCA molecules. (a-3) The AFM image recorded in aqueous environment showed that the 2D PFCA nanosheets had a uniform thickness of $3.8 \pm 0.12$ $\mathrm{nm}$; the vertical scale is $42 \mathrm{~nm}$. (b-1 and 2) Schematic formation of spherical BCP micelles in a THF and $\mathrm{H}_{2} \mathrm{O}$ solvent mixture at room temperature and the corresponding cryo-TEM image. (c-1 and 2) Sketch model and cryo-TEM image of the hierarchical supramolecular co-assemblies originating from BCP monolayer arrangement on both surfaces of PFCA nanosheets. (Scale bars, $100 \mathrm{~nm})$

Meanwhile, amphiphilic PS- $b$-PEO diblocks can self-assemble into spherical micelles in a $\mathrm{THF} / \mathrm{H}_{2} \mathrm{O}$ co-solvent system at room temperature. ${ }^{[9]}$ Cryo-TEM revealed the formation of narrowly dispersed spherical micelles of $\mathrm{PS}_{117}-b-\mathrm{PEO}_{114}$ with an average diameter of the PEO cores of approximately $18 \mathrm{~nm}$ (Fig. 1b-2). After mixing of $\mathrm{C}_{18}$-PFCA and $\mathrm{PS}_{117}-b-\mathrm{PEO}_{114}$ micelle solutions, hierarchical supramolecular co-assembly occurred, generating monolayer close-packed micelle arrays of BCP on both sides of the PFCA lamellas (Fig. 1C-2). The driving force of this coassembly process is considered to be hydrogen bonding between the PEO coronae of the micelles and carboxylic acid groups $(-\mathrm{COOH})$ of PFCA, which promotes the effective adsorption and secondary self-assembly of BCP micelles on the PFCA lamella surfaces. This process was verified by the fluid AFM, which displayed the close packing of spherical BCP micelles on the PFCA nanosheets with a uniform thickness of approximately $45 \mathrm{~nm}$ (Supplementary Fig. 3), close to twice the diameter of $\mathrm{PS}_{117}-b-\mathrm{PEO}_{114}$ micelles, which supports the finding of monolayer self-assembly of BCP micelles on both sides of the PFCA nanosheets.

The aforementioned 2D hierarchical co-assemblies can confine the polymerization of aniline monomers into mesoscale-ordered conducting polymer nanosheets. Therefore, aniline and ammonium persulfate were added at room temperature (see the experimental section), triggering the formation of polymeric networks around the PEO domains of the tightly packed BCP micelles (Fig. a-1). The self-assembled PFCA nanosheets here ensure the formation of $2 \mathrm{D}$, free-standing architecture, whereas the spherical BCP micelles serve as pore-introducing moieties to establish a mesoscale porous structure. After removal of the BCP and PFCA templates by extraction with THF and ethanol, 2D conducting polymer nanosheets with mesoscale ordering were obtained (Fig. a-2). Fourier-transform infrared (FT-IR) spectroscopy clearly confirmed the complete removal of the block copolymers and PFCA lipids, evidenced by the disappearance of the characteristic signals of PS- $b$-PEO and the $\mathrm{C}-\mathrm{F}$ vibrations of PFCA (Supplementary Fig. 4). Figure 2 shows the structural characterization of the mPANI nanosheets synthesized from $\mathrm{PS}_{117}-b-\mathrm{PEO}_{114}$ and $\mathrm{C}_{18}$-PFCA (mPANI-1, 2 and 3 correspond to $\mathrm{PS}_{49}-b-\mathrm{PEO}_{114}, \mathrm{PS}_{117}-b-\mathrm{PEO}_{114}$ and $\mathrm{PS}_{146^{-}}$ $b$-PEO ${ }_{114}$, respectively). The scanning electron micrograph (SEM, Fig. 2b) reveals that the mPANI-2 nanosheets derived from $\mathrm{PS}_{117}-b$ - $\mathrm{PEO}_{114}$ and $\mathrm{C}_{18}$-PFCA possess regular arrays of pores with a uniform size of approximately $15 \mathrm{~nm}$. Interestingly, the AFM image in Figure $2 \mathrm{c}$ indicates that the mPANI-2 nanosheets have a flat surface with a thickness of about $15 \mathrm{~nm}$, which is the same size as the core diameter of the $\mathrm{PS}_{117}-b$ $\mathrm{PEO}_{114}$ micelles. This result suggests that the template removal resulted in exfoliation of the sandwich-structured mPANI/template/mPANI, generating monolayer mPANI nanosheets (as illustrated in Fig. 2a-2).

The TEM examination (Fig. 2d) confirms the well-ordered, hexagonally mesoporous structure of the mPANI-2 nanosheets with a uniform pore size. The corresponding fast Fourier transform (FFT) exhibits a hexagonal pattern, indicative of lateral ordering, with a domain size greater than $600 \mathrm{~nm}$ in the mesoporous mPANI nanosheets. GISAXS was further employed to determine the periodic mesostructures of the mPANI nanosheets (Fig. $2 \mathrm{e}$ ), showing a diffraction on the equator with $d$-spacing of about $30 \mathrm{~nm}$, indexed to the 100 reflection of $2 \mathrm{D}$ hexagonal structure with a lattice parameter $a_{h e x}=34.7 \mathrm{~nm}$. This 
result agrees well with the TEM and FFT examinations of the ordered structure of the mPANI nanosheets at the mesoscale level.

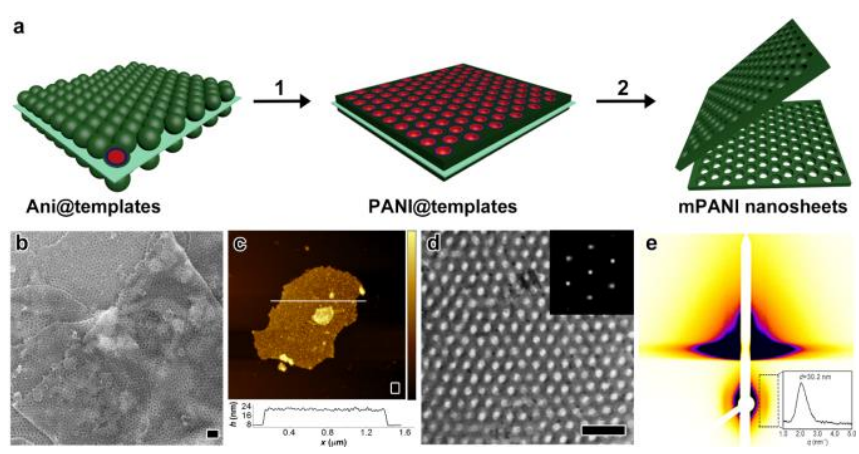

Figure 2. Formation of $2 \mathrm{D}$ mesoscale-ordered conducting polymer nanosheets. (a-1) Polymerization of aniline monomers (dark green) directed by the hierarchical supramolecular co-assemblies of spherical BCP micelles (red and blue) and PFCA nanosheet (light green) templates. (a-2) Formation of 2D mesoscale-ordered conducting polymer monolayers after the removal of the BCP and PFCA templates. (b) SEM image of the mPANI-2 nanosheets. (c) AFM image of mPANI-2 nanosheets (Vertical scale is $44 \mathrm{~nm}$ ). (d) The TEM image and fast Fourier transform (FFT, inset) show the ordered 2D mesostructured of a mPANI-2 nanosheet with a hexagonal lattice. (e) Radial scan along the equator of the GISAXS results confirms the ordered structure of mPANI-2 nanosheets. (Scale bars, $100 \mathrm{~nm}$ ).

Benefiting from the controllability of the BCP and PFCA templates, the mesostructures of the mPANI polymer nanosheets can be readily controlled with various pore sizes and thicknesses by simply varying the PS block lengths. Figure $3 a$ and Supplementary Table 1 show that the pore size of mPANI-1 decreased to approximately $7 \mathrm{~nm}$ with a thickness of approximately $13 \mathrm{~nm}$, when using the $\mathrm{PS}_{49}-b-\mathrm{PEO}_{114}$ with a shorter PS block as the template. In contrast, with the PS block length increased to 146, the pore size of mPANI-3 nanosheets increases to approximately $18 \mathrm{~nm}$ and the thickness reaches approximately $27 \mathrm{~nm}$ (Fig. 3b). In addition to controlling the pore size and thickness, the PS block length plays a crucial role in governing the Brunauer-Emmett-Teller (BET) surface area and pore volume of the mesoporous nanosheets. The $\mathrm{N}_{2}$ sorption isotherms of the mPANI nanosheets exhibit a type IV curve with an $\mathrm{H}_{2}$-type hysteresis loop (Supplementary Fig. 5 and Table 1). The specific surface area and pore volume of the mPANI-2 nanosheets are $85 \mathrm{~m}^{2} \mathrm{~g}^{-1}$ and $0.2 \mathrm{~cm}^{3} \mathrm{~g}^{-1}$, respectively, which exceed those of the previously reported PANI materials (Supplementary Table 2). Moreover, the specific surface area of the mPANI-2 nanosheets is larger than that of the mPANI-1 nanosheets $\left(25 \mathrm{~m}^{2} \mathrm{~g}^{-1}\right)$ because a longer PS block results in larger pores and completely perforated channels in the nanosheets, leading to enhanced specific surface area of the mPANI nanosheets. Nevertheless, the oversized pores in the case of mPANI-3 nanosheets result in a decreased specific surface area $\left(33 \mathrm{~m}^{2} \mathrm{~g}^{-1}\right)$.

The TEM images of the mPANI-1 and mPANI-3 nanosheets reveal their well-defined, hexagonally mesoporous structures with uniform pore sizes (Fig. 3). The inset 2D FFT of the hexagonal pattern indicates lateral mesoscale ordering within the mPANI-1 nanosheets (Fig. 3a). Additionally, the 2D FFT of the mPANI-3 nanosheets displays several sets of hexagonal patterns, indicating a relatively lower regularity of mPANI-3. The GISAXS images of the mPANI-1 and mPANI-3 nanosheets show no diffractions on the equator, indicating that they lack long-range mesoscale ordering within the nanosheet structures (Supplementary Fig. 6).

On the other hand, by choosing PFCA templates with variable hydrophobic chain lengths, we can further control the morphology of the mPANI nanosheets from large, 13-nm-thick monolayers to 45-nm-thick, ribbon-like double layers (Supplementary Fig. 7). The TEM study shows that both of the mPANI nanosheets obtained by using $\mathrm{C}_{12}$-PFCA and $\mathrm{C}_{14}$-PFCA templates have mesoporous, ribbon-like structures (Supplementary Fig. 7). In contrast to the $\mathrm{C}_{14^{-}} \mathrm{PFCA}$ or $\mathrm{C}_{18^{-}}$ PFCA templates, which yield monolayer features, the TEM characterization of the mPANI nanosheets derived from $\mathrm{C}_{12-}$ PFCA and PS $_{117}-b-$ PEO $_{114}$ templates shows a double-layer stacking structure, which hold a thickness of approximately 45 $\mathrm{nm}$, according to AFM measurements. We hypothesize that $\mathrm{C}_{14}$ PFCA or $\mathrm{C}_{18}$-PFCA with longer hydrophobic chains, lead to larger spaces between two PANI layers and a weaker connection between polymeric backbones in the sandwich structure, thereby facilitating detachment into monolayers (Fig. 2a-2).
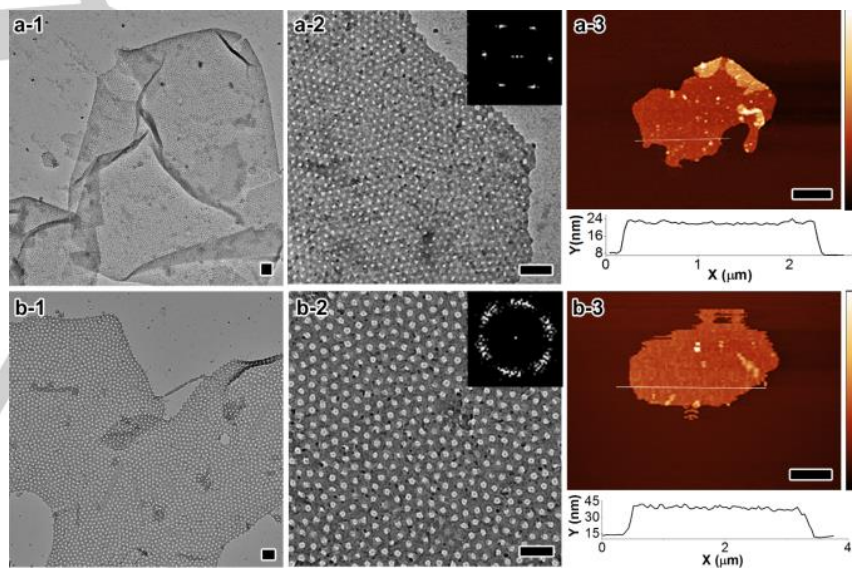

Figure 3. Structure control of the free-standing mesoporous conducting polymer nanosheets. (a-1, 2) TEM images, fast Fourier transform (FFT, inset) and (a-3) AFM of mPANI nanosheets synthesized with $\mathrm{PS}_{49}-b-\mathrm{PEO}_{117}$ and $\mathrm{C}_{18^{-}}$ PFCA. (b-1, 2) TEM images, FFT (inset) and (b-3) AFM of mPANI nanosheets synthesized with $\mathrm{PS}_{146}-b-\mathrm{PEO}_{117}$ and $\mathrm{C}_{18}-\mathrm{PFCA}$. Scale bar for TEM, $100 \mathrm{~nm}$; vertical scale for AFM, $61 \mathrm{~nm}(\mathrm{a}-3)$ and $103 \mathrm{~nm}(\mathrm{~b}-3)$.

Considering the strong confinement effect of the hydrogen bonding and electrostatic interactions between the aniline monomers and BCP/PFCA templates, the compact arrangement of the resulting linear PANI backbone in the nanosheet structures strongly affects the electrical conductivity of PANI. ${ }^{[10]}$ Thus, we used $X$-ray diffraction (XRD) to investigate the polymeric backbone arrangement within the mPANI nanosheets. For comparison, we synthesized PANI samples under the same conditions but without using a template. The resulting sample contained only irregular PANI nanoparticles (denoted as PANI- 
blank, Supplementary Fig. 8). Two peaks centered at approximately $2 \theta=20^{\circ}$ and $25^{\circ}$, corresponding to a $d$-spacing of 4.4 and $3.6 \AA$, respectively, were observed in the XRD patterns of the PANI-blank samples (Fig. 4a). These two peaks are generally ascribed to periodicity parallel and perpendicular to the polymer backbone. ${ }^{[11]}$ Notably, the latter peak of the mPANI nanosheets shows an apparent shift to the higher diffraction angle $\left(2 \theta=30.4^{\circ}\right.$, corresponding to a $d$-spacing of $\left.3.0 \AA\right)$ along with higher relative intensity (Fig. $4 \mathrm{a})$. This result suggests that mPANI nanosheets have stronger $\pi-\pi$ stacking interactions in the polyaniline backbone than in PANI-blank samples. ${ }^{32}$

\begin{tabular}{lccc}
\hline Samples & $\begin{array}{c}\text { Conductivity } \\
\text { of films }\end{array}$ & $\begin{array}{c}\text { Vertical } \\
\text { conductivity }^{\text {of films }}{ }^{[a]}\end{array}$ & $\begin{array}{c}\text { Vertical } \\
\text { conductivity of } \\
\text { monolayers }^{[\mathrm{b}]}\end{array}$ \\
\hline PANI-blank film & 12 & - & - \\
mPANI-2 as-made film & 41 & $3.3 \times 10^{-7}$ & $4.6 \times 10^{-3}$ \\
mPANI-2 film (1 M HCl) & 116 & $5.9 \times 10^{-7}$ & $4.7 \times 10^{-2}$ \\
mPANI-2 film $(2 \mathrm{M} \mathrm{HCl})$ & 188 & $1.3 \times 10^{-6}$ & 1.3 \\
\hline
\end{tabular}

Note: (a) The film samples were prepared from the corresponding mPANI-2 nanosheets by filtration (see method in the experiment). (b) As obtained by measurement of the single mPANI-2 nanosheet.
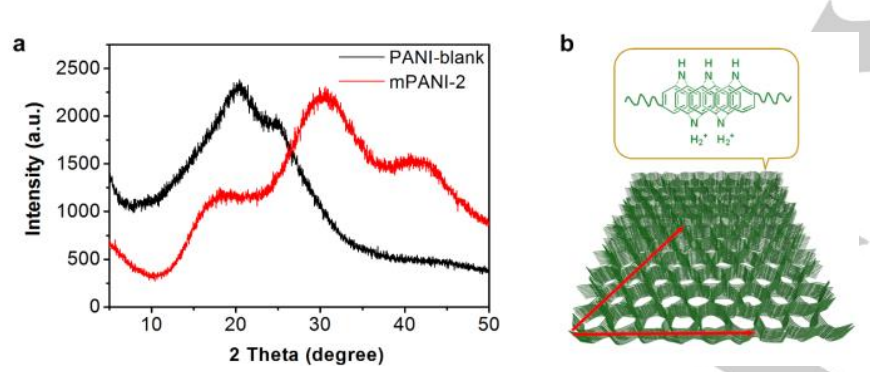

Figure 4. Conductivity of the $2 \mathrm{D}$ mesoscale-ordered conducting polymer nanosheets. (a) XRD patterns of the PANI control sample (PANI-blank) and as-made mPANI-2 nanosheets. (b) Scheme of the densely stacked benzene rings in the compact mPANI polymeric linear backbones. The red arrows indicate that the in-plane electron interactions are easier than those of the vertical direction, thereby contributing the majority of the electrical conductivity of the mPANI nanosheets.

A standard four-point-probe measurement on the filtrated films of the mPANI-2 nanosheets was subsequently performed. As shown in Table 1, the approximately $7.96 \mu$ m-thick mPANI-2 films prepared by the filtration of the mPANI-2 nanosheets (Supplementary Fig. 8) exhibited an exceptionally high electrical conductivity of $41 \mathrm{~S} \mathrm{~cm}^{-1}$, which is the highest reported value for pristine PANI conducting polymers (Supplementary Table 3). ${ }^{[10 \mathrm{~b}}$, 12] Additionally, the lower electrical conductivity of the PANIblank films (12 $\mathrm{S} \mathrm{cm}^{-1}$, Table 1) also highlights that the $2 \mathrm{D}$ mPANI nanosheets with stronger $\pi-\pi$ stacking interactions in the polyaniline backbone are essential for the enhanced electron transport behavior. ${ }^{32}$

Moreover, the unique 2D architecture provides the mPANI nanosheets with anisotropic conductivity along the in- and out- of-plane directions. We further evaluated the vertical conductivity of the as-prepared mPANI-2 films (see method in the Supplementary experiment). The results show that the vertical conductivity of the mPANI-2 films is approximately $3.3 \times 10^{-7} \mathrm{~S}$ $\mathrm{cm}^{-1}$ at room temperature (Table 1), which is much lower than the electrical conductivity $\left(41 \mathrm{~S} \mathrm{~cm}^{-1}\right)$ of the film measured by the four-point-probe method. To avoid the interference of the barrier resistance of the mPANI layers in the films, we also used current-sensing AFM (CS-AFM) to test the vertical conductivity of a single mPANI-2 nanosheet. The CS-AFM analysis reveals a vertical conductivity of approximately $4.6 \times 10^{-3} \mathrm{~S} \mathrm{~cm}^{-1}$ for the mPANI-2 monolayer. These results indicate that mPANI nanosheets have anisotropic electrical conductivity with superiority along the in-plane direction (Fig. 4 b).

The mPANI-2 films were further doped with $1 \mathrm{~mol} \mathrm{~L}^{-1}$ hydrochloric acid $(\mathrm{HCl})$, and the electrical conductivity of the mPANI-2 films reached $116 \mathrm{~S} \mathrm{~cm}^{-1}$ and further increased to 188 $\mathrm{S} \mathrm{cm}^{-1}$ when doped with $2 \mathrm{~mol} \mathrm{~L}^{-1} \mathrm{HCl}$. Similarly, both the single mPANI-2 nanosheet and the film samples showed improved vertical conductivity after $\mathrm{HCl}$ doping (Table 1). The remarkable increase in electrical conductivity of the doped mPANI nanosheets can be ascribed to additionally generated electronic states and charge defects induced by protonic acid doping. ${ }^{[13]}$ In summary, by manipulating two types of typical micellar assemblies of BCP and PFCA in different dimensions, we successfully demonstrated an unprecedented and elegant approach for producing 2D free-standing mesoscale-ordered conducting polymer nanosheets via simple solution-based controlled supramolecular self-assembly. The resulting 2D ultrathin mesoporous conducting polymer materials are characterized by ordered hexagonal pore arrays, tunable morphologies and pore sizes, and large specific surface area. This unique template-directed polymerization of PANI around BCP micelles produces densely $\pi-\pi$ stacked linear polymer backbone, thereby endowing the mPANI nanosheets with anisotropic and record-high electrical conductivity. The selfassembly fabrication of ordered mesoporous conducting polymers still remains a great challenge up to date, although the first mesoporous materials was discovered over more than two decades ago. Hence, by successful incorporation of mesoscale ordering into conducting polymer nanosheets, we provided here new insights into multicomponent co-assembly systems that resulted in a new member to the mesoporous material as well as 2D material family. The conducting polymer nanosheets produced by solution-based approach also open new opportunities for the development of low-cost processing techniques towards flexible electronics, sensors and energy devices.

\section{Acknowledgements}

This work was financially supported by the ERC Grant 2DMATER, EC under Graphene Flagship (no. CNECT-ICT604391), the DFG via EXC1056, the 973 Program of China (2012CB933404 and 2013CB328804), the Natural Science Foundation of China (21320102006, 21304057 and 51573091), the Program for Professor of Special Appointment in Shanghai 
(Eastern Scholar), A.H. acknowledges funding from the EU through the ERC grant NUCLEOPOLY and the STREP project MICreagents, NWO through the Vici Grant and the Zernike Institute for Advanced Materials. We thank Feng Tian (Shanghai Synchrotron Radiation Facility, SSRF, Shanghai), Kai Ke (Leibniz Institute for Polymer Research, IPF, Dresden), also thank the cfaed (Center for Advancing Electronics Dresden) and Dresden Center for Nanoanalysis (DCN).

Keywords: two-dimensional $•$ mesoporous $•$ conducting polymer - polyaniline $\cdot$ self-assembly

[1] a) K. S. Novoselov, A. K. Geim, S. Morozov, D. Jiang, Y. Zhang, S. a Dubonos, I. Grigorieva, A. Firsov, Science 2004, 306, 666-669; b) S. Stankovich, D. A. Dikin, G. H. Dommett, K. M. Kohlhaas, E. J. Zimney, E. A. Stach, R. D. Piner, S. T. Nguyen, R. S. Ruoff, Nature 2006, 442, 282-286; c) K. Novoselov, A. K. Geim, S. Morozov, D. Jiang, M. Katsnelson, I. Grigorieva, S. Dubonos, A. Firsov, Nature 2005, 438 197-200; d) M. Chhowalla, H. S. Shin, G. Eda, L.-J. Li, K. P. Loh, H. Zhang, Nat Chem 2013, 5, 263-275; e) X. Peng, L. Peng, C. Wu, Y. Xie, Chem. Soc. Rev. 2014, 43, 3303-3323; f) C. Tan, H. Zhang, Chem. Soc. Rev. 2015, 44, 2713-2731.

[2] a) X. Li, X. Wang, L. Zhang, S. Lee, H. Dai, Science 2008, 319, 1229 1232; b) Y. Hernandez, V. Nicolosi, M. Lotya, F. M. Blighe, Z. Sun, S. De, I. McGovern, B. Holland, M. Byrne, Y. K. Gun'Ko, Nature Nanotech. 2008, 3, 563-568; c) F. Bonaccorso, L. Colombo, G. Yu, M. Stoller, V. Tozzini, A. C. Ferrari, R. S. Ruoff, V. Pellegrini, Science 2015, 347, 1246501-1246506; d) X. Li, W. Cai, J. An, S. Kim, J. Nah, D. Yang, R Piner, A. Velamakanni, I. Jung, E. Tutuc, Science 2009, 324, 13121314; e) J. N. Coleman, M. Lotya, A. O'Neill, S. D. Bergin, P. J. King, U. Khan, K. Young, A. Gaucher, S. De, R. J. Smith, Science 2011, 331, $568-571$; f) M. R. Lukatskaya, O. Mashtalir, C. E. Ren, Y. Dall'Agnese P. Rozier, P. L. Taberna, M. Naguib, P. Simon, M. W. Barsoum, Y. Gogotsi, Science 2013, 341, 1502-1505; g) S. Najmaei, Z. Liu, W. Zhou, X. Zou, G. Shi, S. Lei, B. I. Yakobson, J.-C. Idrobo, P. M. Ajayan, J. Lou, Nat. Mater. 2013, 12, 754-759.

[3] a) N. R. Champness, Nat Chem 2014, 6, 757-759; b) J. W. Colson, W. R. Dichtel, Nat Chem 2013, 5, 453-465; c) M. J. Kory, M. Wörle, T. Weber, P. Payamyar, W. van de PollStan, J. Dshemuchadse, N. Trapp, A. D. Schlüter, Nat Chem 2014, 6, 779-784; d) X. Zhuang, Y. Mai, D. Wu, F. Zhang, X. Feng, Adv. Mater. 2014, 27, 403-427.

[4] a) H. Sai, K. W. Tan, K. Hur, E. Asenath-Smith, R. Hovden, Y. Jiang, M. Riccio, D. A. Muller, V. Elser, L. A. Estroff, Science 2013, 341, 530 534; b) C. Poelking, M. Tietze, C. Elschner, S. Olthof, D. Hertel, B. Baumeier, F. Würthner, K. Meerholz, K. Leo, D. Andrienko, Nat. Mater. 2015, 14, 434-439; c) M. B. Ross, J. C. Ku, V. M. Vaccarezza, G. C. Schatz, C. A. Mirkin, Nature Nanotech. 2015, 10, 453-458; d) J. Liu, N. P. Wickramaratne, S. Z. Qiao, M. Jaroniec, Nat Mater 2015, 14, 763774.

[5] a) B. Kong, J. Tang, Y. Zhang, T. Jiang, X. Gong, C. Peng, J. Wei, J. Yang, Y. Wang, X. Wang, G. Zheng, C. Selomulya, D. Zhao, Nat Chem 2016, 8, 171-178; b) Y. Fang, Y. Lv, R. Che, H. Wu, X. Zhang, D. Gu, G. Zheng, D. Zhao, J. Am. Chem. Soc. 2013, 135, 1524-1530.

[6] a) S. Liu, P. Gordiichuk, Z.-S. Wu, Z. Liu, W. Wei, M. Wagner, N. Mohamed-Noriega, D. Wu, Y. Mai, A. Herrmann, K. Müllen, X. Feng, Nat Commun 2015, 6, 8817; b) J. Mahmood, E. K. Lee, M. Jung, D. Shin, H.-J. Choi, J.-M. Seo, S.-M. Jung, D. Kim, F. Li, M. S. Lah, Proc. Natl. Acad. Sci. U. S. A. 2016, 201605318.

[7] a) O. Bubnova, Z. U. Khan, A. Malti, S. Braun, M. Fahlman, M. Berggren, X. Crispin, Nat. Mater. 2011, 10, 429-433; b) J. r. Heinze, B.

A. Frontana-Uribe, S. Ludwigs, Chem. Rev. 2010, 110, 4724-4771; c) J.

E. Yoo, K. S. Lee, A. Garcia, J. Tarver, E. D. Gomez, K. Baldwin, Y. Sun, H. Meng, T. Q. Nguyen, Y. L. Loo, Proc. Natl. Acad. Sci. U. S. A 2010, 107, 5712-5717.

[8] a) G. Yu, K. Jie, F. Huang, Chem. Rev. 2015, 115, 7240-7303; b) T. Shimizu, M. Masuda, H. Minamikawa, Chem. Rev. 2005, 105, 14011444.

[9] Y. Mai, A. Eisenberg, Chem. Soc. Rev. 2012, 41, 5969-5985

[10] a) G. Giri, E. Verploegen, S. C. Mannsfeld, S. Atahan-Evrenk, D. H. Kim, S. Y. Lee, H. A. Becerril, A. Aspuru-Guzik, M. F. Toney, Z. Bao, Nature 2011, 480, 504-508; b) L. Pan, G. Yu, D. Zhai, H. R. Lee, W. Zhao, N. Liu, H. Wang, B. C.-K. Tee, Y. Shi, Y. Cui, Z. Bao, Proc. Natl. Acad. Sci. U. S. A. 2012, 109, 9287-9292.

[11] J. P. Pouget, M. E. Jozefowicz, A. J. Epstein, X. Tang, A. G. MacDiarmid, Macromolecules 1991, 24, 779-789.

[12] a) J. Huang, R. B. Kaner, J. Am. Chem. Soc. 2004, 126, 851-855; b) C. Lu, T. Ben, S. Xu, S. Qiu, Angew. Chem. Int. Ed. 2014, 126, 6572-
6576; c) I. Y. Choi, J. Lee, H. Ahn, J. Lee, H. C. Choi, M. J. Park, Angew. Chem. Int. Ed. 2015, 54, 10497-10501.

[13] A. G. MacDiarmid, A. J. Epstein, Faraday Discuss. Chem. Soc. 1989 88, 317-332.

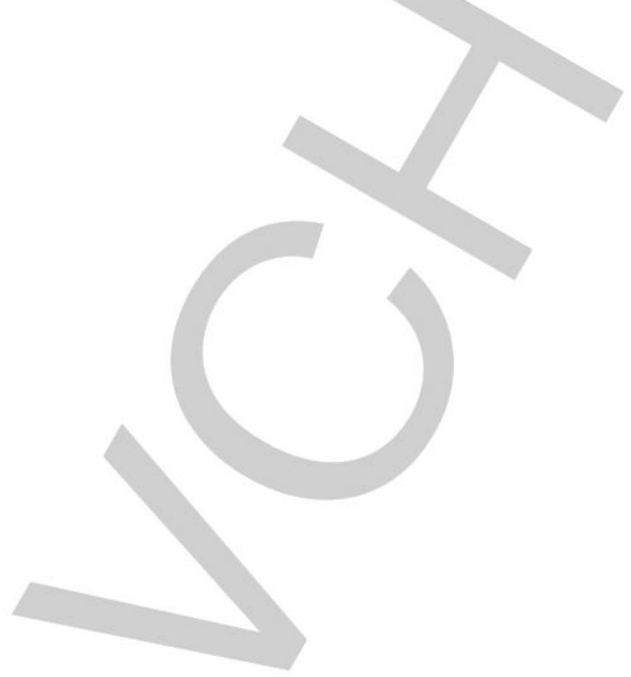




\section{COMMUNICATION}

By synergistically manipulating amphiphilic molecule self-assembly of perfluorocarboxylic acids and polystyrene- $b$-poly(ethylene oxide) diblock copolymers in different dimensions, we successfully achieved 2D ultrathin conducting polymer nanosheets, featuring mesoscaleordered hexagonal pore arrays, tunable morphologies and pore sizes, large specific surface area as well as anisotropic and record-high electrical conductivity.

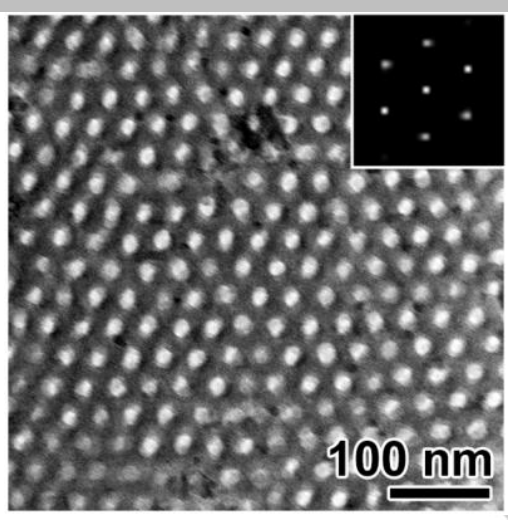

Shaohua Liu, Jian Zhang, Renhao

Dong, Pavlo Gordiichuk, Tao Zhang,

Xiaodong Zhuang, Yiyong Mai, Feng Liu, Andreas Herrmann, and Xinliang Feng*

Page No. - Page No.

Two-Dimensional Mesoscale-Ordered Conducting Polymers 